\title{
Development of Compositional Heterogeneity in Alginate Degraded in Homogeneous Solution
}

\author{
A R N E HA UG, B J ØRN LARSEN, OLAV SMIDSR $\emptyset$ and \\ TEREN CE PAINTER
}

Norwegian Institute of Seaweed Research, N.T.H., Trondheim, Norway

\begin{abstract}
Under conditions that bring about approximately random depolymerisation, samples of sodium alginate were subjected separately, in homogeneous solution, to acid-hydrolysis at $\mathrm{pH} 3.8$, alkaline degradation at $\mathrm{pH} 12$, and oxidative-reductive degradation with a mixture of ascorbic acid and hydrogen peroxide. In all three cases, liberation of discrete polymeric fragments of different composition was indicated by free-boundary electrophoresis before the number-average degree of polymerisation had been reduced to 90 .

It was concluded that the observed behaviour was not due to the presence of weak linkages between differently composed segments of the chains, but was typical of that to be expected of any block copolymer upon random depolymerisation. Studies of composition-distribution in slightly degraded heteropolysaccharides could be generally useful in indicating whether a blockwise arrangement of monomer units is present.
\end{abstract}

U pon degradation at low $\mathrm{pH}$, alginic acid becomes electrophoretically heterogeneous before the number-average degree of polymerisation $\left(\mathrm{P}_{n}\right)$ has been reduced to 440 (Refs. 1, 2). After further degradation three types of fragment are readily separable by fractional precipitation, and relative to the starting-material they are much enriched, respectively, in mannuronicacid residues, in guluronic-acid residues, and in sequences of alternating mannuronic- and guluronic-acid residues.., ${ }^{2,3}$ Examination of these fragments has led to the formulation of alginate as a block copolymer, in which long blocks of contiguous mannuronic-acid residues and similar blocks of guluronicacid residues are joined through regions of the molecule in which the two monomeric units are arranged in a largely alternating sequence..$^{1-4}$

Recently, blockwise arrangements of monosaccharide residues have also been found in dermatan sulphate, ${ }^{5}$ in heparitin sulphate ${ }^{6}$ and in a galacturonorhamnan belonging to the pectic group of polysaccharides, ${ }^{7}$ and it begins to appear that such structures may be quite widespread in Nature. It is therefore of some interest to establish more clearly the nature of the

Acta Chem. Scand. 23 (1969) No. 9 
changes that lead to the early appearance of compositional heterogeneity * when alginate is heated in acid, as this could indicate whether similar methods might be successfully applied to other heteropolysaccharides. The present paper attempts to do this, and also discusses some problems that arise in the nomenclature of heteropolysaccharides that are subject to degradation during isolation.

\section{THEORY}

The random depolymerisation of binary, linear, block copolymers whose structures conform to the ultimate-unit, penultimate-unit and penpenultimateunit models of addition copolymerisation has been treated in detail elsewhere, ${ }^{9,10}$ and formulae permitting extension of the treatment to a simple case of non-random depolymerisation have been developed. ${ }^{4}$ It is now reasoned that the conclusions drawn ${ }^{9,10}$ from these quantitative treatments with defined theoretical models are qualitatively of quite general validity.

This may be illustrated by considering the hypothetical block copolymer depicted below:

$$
[-\mathrm{A}-\mathrm{A}-\mathrm{A}-\mathrm{A}-\mathrm{A}-\mathrm{B}-\mathrm{B}-\mathrm{B}-\mathrm{B}-\mathrm{B}-]_{n}
$$

From one repeating unit of this copolymer, there are five different ways of splitting out a hexamer containing units of both $\mathrm{A}$ and $\mathrm{B}$, but no way of obtaining one composed of A only or of B only. During random depolymerisation, therefore, all fragments larger than pentamers are heteropolymeric. For the pentamers, there is one way of obtaining one composed of A only, one way of obtaining one composed of $\mathrm{B}$ only, but 4 ways of obtaining a pentamer of mixed composition. Continuation of this reasoning leads to the figures shown in Table 1.

Table 1. Number of ways of choosing an $n$-mer composed of A only, an $n$-mer composed of $\mathrm{B}$ only, and an $n$-mer of mixed composition from a segment of a copolymer chain consisting of five consecutive units of $\mathrm{A}$ and five of $\mathrm{B}$.

\begin{tabular}{cccc}
$n$ & A only & B only & A and B \\
\hline 6 & 0 & 0 & 5 \\
5 & 1 & 1 & 4 \\
4 & 2 & 2 & 3 \\
3 & 3 & 3 & 2 \\
2 & 4 & 4 & 1 \\
1 & 5 & 5 & 0 \\
\hline
\end{tabular}

It is evident that, as depolymerisation proceeds, the probability of finding a homopolymeric fragment in the reaction mixture increases, while that of finding a heteropolymeric fragment decreases. Eventually, a stage is reached

* Throughout this paper, the terms "heterogeneous" and "homogeneous" are used in the sense defined by Gibbons," who distinguishes them clearly from "polydispersity" and "monodispersity", respectively. Some minor qualifications to Gibbons' nomenclature have been discussed by the present authors. ${ }^{9}$

Acta Chem. Scand. 23 (1969) No. 9 
at which a physical method that separates molecules upon a basis of composition will demonstrate the presence of more than one discrete component. The longer the blocks, the earlier will such heterogeneity appear. If the blocks composed of units of $A$ are longer than those composed of units of $B$, homopolymeric fragments composed of A only will appear as a discrete entity in the reaction mixture at a lower degree of scission than will fragments composed of $\mathrm{B}$ only.

Extension of this reasoning to other hypothetical cases readily permits the conclusion that it is not necessary that there be only two kinds of block, or that they be all of the same length, or that they be homopolymeric. Compositionally discrete blocks must always appear as compositionally discrete fragments at a stage in the depolymerisation which is determined by their length.

\section{EXPERIMENTAL}

Material and methods. The sample of sodium alginate was prepared from Laminaria digitata (Tarva 29/8), and analysis by the method of Haug and Larsen ${ }^{11}$ indicated the presence of mannuronic-acid residues $(61.5 \%)$ and guluronic-acid residues $(38.5 \%)$. Free-boundary electrophoresis was carried out as described previously ${ }^{2}$ in a medium consisting of sodium chloride $(0.05 \mathrm{M})$ and calcium chloride $(0.00075 \mathrm{M})$. Number-average degrees of polymerisation $\left(\mathrm{P}_{n}\right)$ were determined by the measurement of reducing power with Nelson's method, ${ }^{12}$ using mannuronic acid as the standard. For alkaline degradation, $\mathrm{P}_{n}$ was determined by the thiobarbituric-acid reaction, ${ }^{13}$ which had previously been calibrated ${ }^{14}$ against the Nelson method.12

Hydrolysis at $p H 3.8$. Alginate $(0.5 \% \mathrm{w} / \mathrm{v})$ in citrate buffer $(\mathrm{pH} 3.8 ; 0.025 \mathrm{M})$ was heated under reflux at $100^{\circ}$, and at intervals samples were withdrawn, cooled, and neutralised with sodium bicarbonate.

Oxidative-reductive degradation..$^{15}$ Aqueous alginate $(1 \% \mathrm{w} / \mathrm{v})$ was mixed with an equal volume of a solution of ascorbic acid $(0.02 \mathrm{M})$ in sodium bicarbonate $(0.2 \mathrm{M})$ at room temperature. An equal volume of hydrogen peroxide $(0.1 \mathrm{M})$ was then added, and the mixture was stirred at room temperature until all the hydrogen peroxide had been consumed, after which a portion of the reaction mixture was removed and dialysed against water prior to analysis. Another equal volume of hydrogen peroxide (0.1 M) was then added, and when it had been consumed, a final sample was dialysed and subjected to analysis.

Alkaline degradation. ${ }^{14}$ The reaction mixture consisted of alginate $(0.5 \% \mathrm{w} / \mathrm{v})$ in $0.5 \mathrm{M}$ tripotassium phosphate $(\mathrm{pH} 12.0)$, to which potassium borohydride $(0.2 \% \mathrm{w} / \mathrm{v})$ was added to inhibit the alkaline peeling reaction. ${ }^{16}$ It was heated at $100^{\circ}$, and samples were withdrawn at intervals, cooled, and neutralised with acetic acid.

Fractional precipitation. Alginate degraded for $3 \mathrm{~h}$ at $100^{\circ}$ and $\mathrm{pH} 3.9$ (citrate buffer) was fractionally precipitated, first at $\mathrm{pH} 2.7$, and then with calcium ions in the presence of magnesium ions, according to the detailed procedure described previously. ${ }^{2}$ The fractions were reconverted into the sodium form by dialysis, first against sodium ethylenediamine tetracetate and then against water, prior to electrophoresis and analysis.

\section{RESULTS AND DISCUSSION}

Although the considerations in the theoretical section suggest that the early appearance of gross compositional heterogeneity is to be expected when a block copolymer such as alginate is randomly degraded, this is not proof of the absence of especially weak linkages between compositionally distinct segments of the alginate molecules, or that the observed behaviour of alginate ${ }^{1-3}$ is typical of that which can be expected from block copolymers in general.

Acta Chem. Scand. 23 (1969) No. 9 
Linkages may be especially weak in the chemical sense, or when the reaction mixture is not a true solution, they may be effectively weak, relative to linkages that are protected from cleavage by physical factors such as inaccessibility. In the earlier work, ${ }^{1-3}$ depolymerisation was carried out at low $\mathrm{pH}$, under which conditions alginic acid is insoluble, and the results are difficult to interpret in mechanistic terms. In the present work, depolymerisation was effected in homogeneous solutions, and by three different mechanisms, all of which are known to bring about approximately random scission of the chains.

In one experiment, alginate was degraded at $\mathrm{pH} \mathrm{3.8,} \mathrm{at} \mathrm{which} \mathrm{it} \mathrm{is} \mathrm{soluble.}$ The principal degradative mechanism in this case is known to be intramolecular catalysis of the hydrolysis of glycosidic linkages by the carboxyl groups in the respective aglycone units. ${ }^{17}$ Under the conditions used, glycosidic linkages attached at $\mathrm{C}(4)$ to mannuronic-acid residues are cleaved about twice as fast as linkages attached at $\mathrm{C}(4)$ to guluronic-acid residues. Viscosimetric studies of the early stages of degradation gave a linear plot of $\Delta[\eta]^{-1}$ against time, indicating near-random cleavage. ${ }^{18}$ Although insensitive to small differences in the rates of cleavage of the principal glycosidic linkages in the chains, this method should readily have detected the presence of a small proportion of very labile linkages in the chains.

A

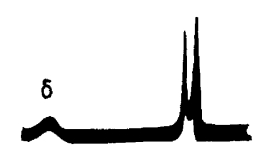

$0.5 h, P_{n}=96$

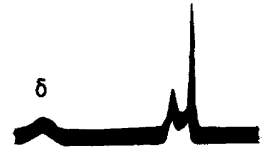

$1.0 \mathrm{~h}, \mathrm{P}_{\mathrm{n}}=55$

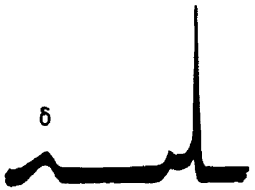

$3.0 \mathrm{~h}, \mathrm{P}_{n}=26$

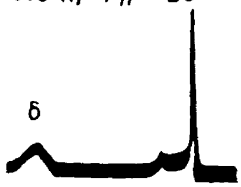

$5.0 \mathrm{~h}, \mathrm{P}_{n}=16$
B

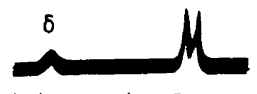

1st sample, $P_{n}=92$

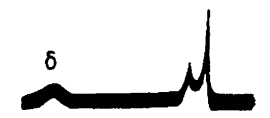

2nd sample, $P_{n}=41$
C

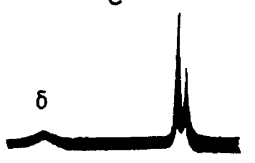

$0.5 \mathrm{~h}, P_{n}=140$

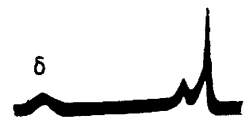

$1.5 \mathrm{~h}, P_{n}=50$

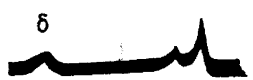

3.5 h, $P_{n}=35$

Fig. 1. Ascending boundaries obtained for alginates degraded at $\mathrm{pH} 3.8$ (A); by oxidativereductive degradation (B); and at $\mathrm{pH} 12$ (C). The stationary boundaries are indicated by $\delta$. 
In another experiment, alginate was degraded at $\mathrm{pH} 12$. The mechanism in this case is a $\beta$-alkoxycarbonyl elimination reaction, and near-random depolymerisation is again indicated by both viscosimetric studies and endgroup analysis. ${ }^{14}$

In the third experiment, alginate was depolymerised by a mixture of ascorbic acid and hydrogen peroxide. The mechanism of this reaction, which belongs to a general class known as "oxidative-reductive degradation" (Ref. 19), is complex and still somewhat obscure, but cleavage of the chain appears to be brought about by hydroxyl radicals ${ }^{20,21}$ and leads to the liberation of reducing end-groups. ${ }^{22,23}$ Random depolymerisation is again indicated by viscosimetric studies ${ }^{20-23}$ and reducing end-group determinations. ${ }^{23}$ As pointed out earlier, ${ }^{23}$ the choice of mannuronic acid as the standard in the $\mathrm{P}_{n}$ determinations is in this case arbitrary, and the absolute values given in Fig. 1 are subject to error.

The results of the three experiments are summarised in Fig. 1, and show a remarkable similarity in the electrophoretic patterns for similar degrees of scission, regardless of the method of degradation. Any attempt to explain them on the basis of "weak linkage" hypotheses would appear to require the assumption that essentially the same linkages are at the same time weak

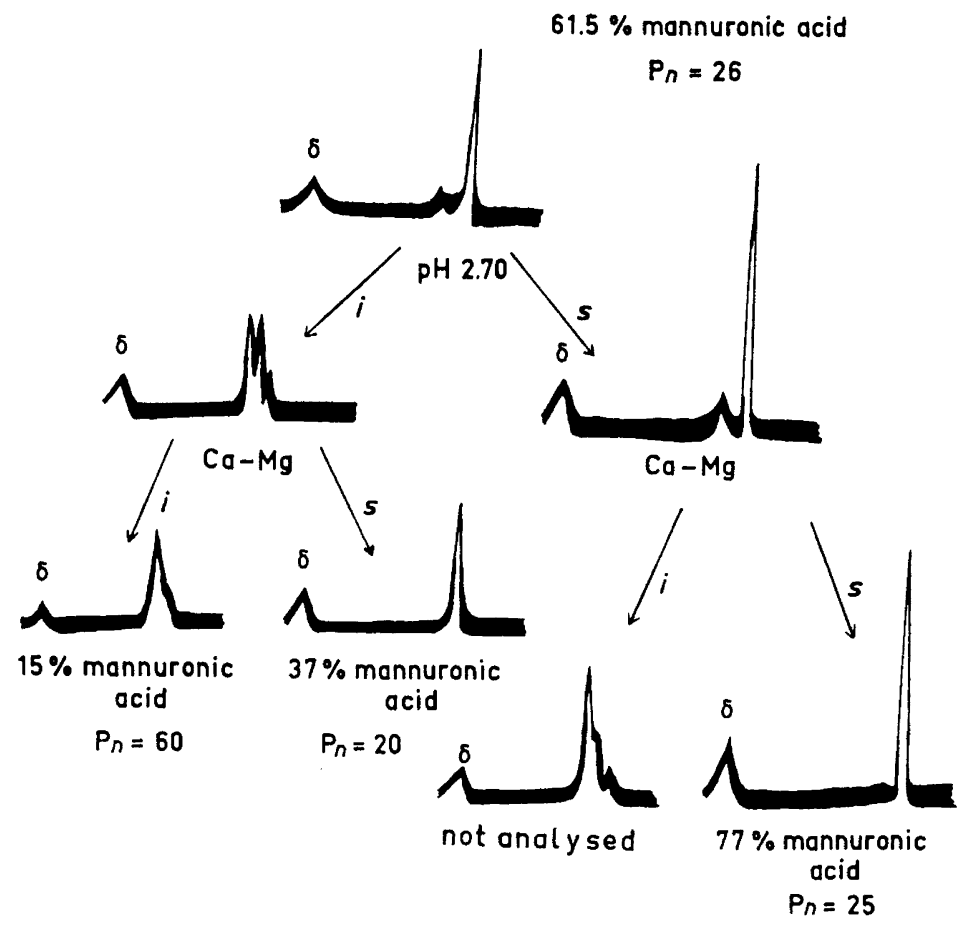

Fig. 2. Ascending boundaries of fractions obtained after degradation of alginate for $3 \mathrm{~h}$ at $100^{\circ}, \mathrm{pH} \mathrm{3.9}$. The method of fractionation is indicated by $p H 2.70$ or $\mathrm{Ca}-\mathrm{Mg}$, the soluble and insoluble fractions by $s$ and $i$, respectively, and the stationary boundaries by $\delta$.

Acta Chem. Scand. 23 (1969) No. 9 
with respect to acid-hydrolysis, alkaline degradation, and oxidative reductive degradation. It would also require that the rate of cleavage of the weak linkages relative to that of ordinary linkages be approximately the same, regardless of the method of degradation.

Thermal and other effects can sometimes give rise to spurious peaks in free-boundary electrophoresis. The electrophoretic medium used in the present work was developed ${ }^{2}$ to separate alginate fragments primarily upon a basis of composition, and rests upon the selective binding of calcium ions by the guluronic-acid residues in the chains. ${ }^{24}$ That the separate peaks represent real, compositionally discrete components was firmly established in the earlier work, ${ }^{2}$ but was nevertheless checked again in the present study. The results for degradation at $\mathrm{pH} 3.9$ are shown in Fig. 2.

It can be concluded that the behaviour of alginate shown in Fig. 1 is a quite authentic example of that to be expected on theoretical grounds for random depolymerisation of a block copolymer, and is not due to structural features which are unique to the alginate molecule. The results reported earlier $^{1-3}$ for degradation at low $\mathrm{pH}$ will be less typical, because then the homopolymeric blocks, once liberated, are highly insoluble and resistant to further hydrolysis, which undoubtedly enhances their yield for a given degree of scission. Electrophoresis will, of course, not necessarily always be the best means of studying composition-distribution, and in the general case, the method chosen will have to be appropriate to the properties of the material.

Since a clear practical example is known of the phenomenon discussed in the theoretical section of this paper and in earlier theoretical treatments, ${ }^{9,10}$ it is pertinent to emphasise the need for caution in interpreting the results of studies of composition-distribution in heteropolysaccharides. It is usually difficult or impossible to avoid some degradation during isolation and purification of a polysaccharide, and it is instructive to consider what the present state of the nomenclature of alginates would be, if this polysaccharide were more easily degraded during isolation than is the case.

As an operational description of degraded preparations of alginates, it would of course be valid and possibly useful to recognise the presence of different components, provided it is understood that their individual compositions will vary widely, depending upon the extent of the degradation, and that when the degradation is slight, they may not be found at all. The principal danger of misinterpretation would seem to lie in the assumption that, since the structures of the separate components are seen to approximate to some kind of easily recognisable ideal (in this case, homopolymeric or alternating), the separate existence of these ideal forms is genetically determined, and the whole preparation can be regarded as a physical mixture of them.

Harsh criticisms have occasionally been made of the work of the early polysaccharide chemists, who, lacking the modern physical methods for characterising polymers, frequently carried out structural studies on materials that were subsequently found to be heterogeneous. It seems fair to point out that the mere discovery of compositional heterogeneity in a preparation of a heteropolysaccharide that was previously thought to be homogeneous is not very meaningful unless the origin of the heterogeneity is also ascertained. 
This latter task may be difficult or impossible to accomplish with complete certainty, but a quest for milder methods of extraction, and for possible variation between preparations from different samples of the same biological material, could be usefully undertaken. In addition, the demonstration that further mild degradation causes the separate components to become more sharply differentiated would be an indication that the differentiation already apparent had been brought about by degradation of some parent macromolecule, having a block type of structure. This last technique has been successfully used in this laboratory in studies of the native state of "fucoidan" in Ascophyllum nodosum. ${ }^{25}$

Finally, recognition must be given to the possibility that the polysaccharide may be actively broken down during the normal metabolism of the organism, either in a stepwise or a random manner, and to the fact that the changes in composition-distribution occurring during stepwise biosynthesis resemble those taking place, in the reverse order, during random depolymerisation. ${ }^{9}$ The exact relationship between stepwise and random processes, insofar as they affect composition-distribution, has been discussed in some detail. ${ }^{9}$ Compositional heterogeneity may therefore arise, in vivo, in heteropolysaccharidic material originating from a single synthetase system. Here again, however, the technique of following the changes in composition-distribution occurring upon random depolymerisation of high molecular-weight fractions could be helpful in tracing the origin of the heterogeneity exhibited by the whole material.

\section{REFERENCES}

1. Haug, A., Larsen, B. and Smidsrød, O. Acta Chem. Scand. 20 (1966) 183.

2. Haug, A., Larsen, B. and Smidsrød, O. Acta Chem. Scand. 21 (1967) 691.

3. Haug, A., Myklestad, S., Larsen, B. and Smidsrød, O. Acta Chem. Scand. 21 (1967) 768 .

4. Larsen, B., Smidsrød, O., Haug, A. and Painter, T. Acta Chem. Scand. 23 (1969) 2375 .

5. Fransson, L. and Rodén, L. J. Biol. Chem. 242 (1967) 4170.

6. Cifonelli, J. A. Carbohyd. Res. 8 (1968) 233.

7. Aspinall, G. O., Gestetner, B., Molloy, J. A. and Uddin, M. J. Chem. Soc. C 1968 2555.

8. Gibbons, R. A. Nature 200 (1963) 665.

9. Painter, T. J., Smidsrød, O., Larsen, B. and Haug, A. Acta Chem. Scand. 22 (1968) 1637.

10. Larsen, B., Painter, T. Haug, A. and Smidsrød, O. Acta Chem. Scand. 23 (1969) 355 .

11. Haug, A. and Larsen, B. Acta Chem. Scand. 16 (1962) 1908.

12. Nelson, N. J. Biol. Chem. 153 (1944) 375.

13. Weissbach, H. and Hurwitz, J. J. Biol. Chem. 234 (1959) 705.

14. Haug, A., Larsen, B. and Smidsrød, O. Acta Chem. Scand. 21 (1967) 2859.

15. Smidsrød, O., Haug, A. and Larsen, B. Acta Chem. Scand. 17 (1963) 1473.

16. Whistler, R. L. and BeMiller, J. N. Advan. Carbohydrate Chem. 13 (1958) 289.

17. Smidsrød, O., Larsen, B., Painter, T. and Haug, A. Acta Chem. Scand. 23 (1969) 1573. 
18. Smidsrød, O., Haug, A. and Larsen, B. Acta Chem. Scand. 20 (1966) 1026.

19. Pigman, W., Rizvi, S. and Holley, H. L. Arthritis Rheumat. 4 (1961) 240.

20. Smidsrød, O., Haug, A. and Larsen, B. Acta Chem. Scand. 17 (1963) 2628.

21. Smidsrød, O., Haug. A. and Larsen, B. Acta Chem. Scand. 19 (1965) 143.

22. Larsen, B. and Smidsrød, O. Acta Chem. Scand. 21 (1967) 552.

23. Smidsrød, O., Haug, A. and Larsen, B. Carbohyd. Res. 5 (1967) 482.

24. Smidsrød, O. and Haug, A. Acta Chem. Scand. 22 (1968) 1989.

25. Larsen, B. and Haug, A. Proc. VIth Intern. Seaweed Symp., Santiago de Compostella 1968. In press.

Received March 18, 1969. 